\title{
THE INCIDENCE OF LIDOCAINE ALLERGY IN DENTISTS: AN EVALUATION OF 100 GENERAL DENTAL PRACTITIONERS
}

\author{
ANNA JANAS-NAZE and PIOTR OSICA \\ Medical University of Lodz, Łódź, Poland \\ Department of Oral Surgery
}

\begin{abstract}
Objectives: Local anesthetics are some of the most common drugs used in dentistry and about 6 million people daily around the world are subjected to their effect in procedures performed by a similar number of dentists who, in turn, are usually not aware of the fact of being allergic to this group of medications. Assuming that dentists are also patients, and that in their daily practice the contact with the allergen is very frequent, it is essential to assess the incidence of allergy to lidocaine in general dental practitioners. Material and Methods: The authors evaluated a group of 100 general dental practitioners in whom adverse reactions similar to anaphylaxis occurred after local anesthesia. The study included individuals who, in their thorough medical history, had experienced episodes of such reactions regarding the skin, airways, gastrointestinal tract and other areas. Results: The relations between type I hypersensitivity and certain symptoms, time from exposure to their appearance, as well as time from the last episode were investigated. Allergy to lidocaine was detected in 17 subjects - type I hypersensitivity was diagnosed in 13 cases (skin prick test - 7; intradermic test - 6) and 4 subjects had IgE-independent allergy (patch test). In the group where type I hypersensitivity was detected, urticaria, angioedema and rhinitis were indicated as inclusion criteria more often than among other subjects. According to the results, the occurrence of angioedema increased the risk of detection of type I hypersensitivity 68.8 times, and 1 year longer period from the last episode decreased this risk by circa $55 \%$. Conclusions: The most important, from the clinical practice point of view, was to show the relation between the medical history indicating the anaphylactic nature of the lidocaine intolerance, and an allergy confirmed by skin tests. Int J Occup Med Environ Health. 2019;32(3):333-9
\end{abstract}

Key words:

allergy, dentists, anaphylaxis, lidocaine, hypersensitivity, local anaesthetics

\section{INTRODUCTION}

Local anaesthetics are some of the most common drugs used in dentistry. Every day about 6 million people around the world are subjected to their effect in procedures performed by a similar number of dentists who, in turn, are usually not aware of the fact of being allergic to this group of medications. The consequences of rare, but serious, adverse reactions after administration of local anaesthetics constitute a major problem in daily general dental practice, primarily due to their common use.
Lidocaine is a common, potent anaesthetic, currently used in almost all types of local anesthesia. Apart from its main use in dentistry, it is also an antiarrhythmic drug, belonging to Class IB according to the Vaughan-Williams Classification of Antiarrhythmic Drugs [1]. It is very effective in controlling premature ventricular contractions, and it shortens the length of action potential and the refractory period [2]. Lidocaine is usually well tolerated; however, its toxicity increases with the concentration of the solution in geometric progression. It can also cause allergic reactions. This drug

Funding: this work was supported by Medical University of Lodz (project No. 501/2-163-01/503-01, project manager: Anna Janas-Naze).

Received: July 5, 2017. Accepted: August 7, 2018.

Corresponding author: Anna Janas-Naze, Medical University of Lodz, Department of Oral Surgery, Pomorska 251, 92-213 Lódź, Poland (e-mail: anna.janas@umed.lodz.pl). 
should not be used in patients with confirmed allergic hypersensitivity to amide local anaesthetics. Also in the case of the second and third degree heart block, severe liver and kidney damage and epilepsy, one should proceed with great caution [3].

The side effects appearing after administering lidocaine are the cause of, very often premature, classification of the patient as "allergic" to local anaesthetics, even though there is no evidence in detailed diagnostic examination. Each adverse reaction after administering the anaesthetic is usually called an allergy and, therefore, every other dose is regarded as a justified concern. In most articles, authors pay attention to the fact that meticulous diagnostics of adverse reactions is mostly aimed at excluding, rather than confirming, the allergy [3,4].

Assuming that dentists are also patients, and that they frequently come into contact with the allergen in their daily practice, it is essential to assess the incidence of allergy to lidocaine in dentists. As the authors were unable to find any reports in the available literature considering occupational allergies to dental anaesthetics, the aim of the study was to investigate the frequency of allergy to locally administered lidocaine in general dental practitioners with histories of adverse reactions to local anaesthetics.

\section{MATERIAL AND METHODS}

The authors evaluated a group of 100 general dental practitioners in whom adverse reactions similar to anaphylaxis occurred after local anesthesia. The study included individuals who, in their thorough medical history, had experienced episodes of such reactions regarding the skin, airways, gastrointestinal tract and other areas. The inclusion criteria were the following symptoms regarding: skin (utricaria, angioedema, erythema), airways (rhinitis, dyspnea), gastrointestinal tract (nausea, vomiting, diarrhoea), and other symptoms such as fainting or vertigo.

Dentists with skin lesions located in the site of performing the diagnostic tests, taking antihistamines and steroids daily, using local steroids and calcineurin inhibitors were excluded from the study group.

The examination was initiated by acquiring medical history information, which included extensive data about the anaphylactic symptoms in the past, which where related to local anesthesia. In the studied group, prick tests with a set of the most common environmental allergens (Allergopharma-Nexter, Poland) and $2 \%$ lidocaine without vasoconstrictors, were performed on the inner part of the forearm. The results were obtained in $20 \mathrm{~min}$. A blister with a diameter above $3 \mathrm{~mm}$ was recognized as a positive result. In the case of a negative or questionable result of the prick test, the studied individual was subjected to intracutaneous testing, which was performed with a tuberculin syringe. The first stage involved the intracutaneous administration of $0.02 \mathrm{ml}$ of lidocaine with a 1 to 1000 dilution (with $0.9 \% \mathrm{NaCl}$ ) and the formation of an initial blister of $3 \mathrm{~mm}$ in diameter. The $0.01 \%$ histamine solution was used as a positive control, and the solution in which the allergenic extract was diluted as a negative control. In the case of no reaction after $20 \mathrm{~min}$, the test was continued by injecting a lidocaine solution with a 1 to 100 dilution. The reaction was also evaluated after $20 \mathrm{~min}$. The test was continued by intracutaneously injecting allergenic solutions with a 1 to 10 dilution, and 1 to 1 in 1 min intervals. The formation of a blister and erythema with a diameter $>5 \mathrm{~mm}$ in the examined area was recognized as a positive result. Patch tests were performed to assess the occurrence of type IV immune response. The investigated substance was 5\% lidocaine gel (Chemotechnique Diagnostics, Sweden). The patch test unit with one of the chambers filled with lidocaine was placed on the upper part of the patient's back. The first reading was performed after $48 \mathrm{~h}$, when the patch test was removed and the patient's skin was examined. The formation of erythema, papules, bumps or blisters in the place where lidocaine had been present was recognized as a positive result. The tests were interpreted 
according to the recommendations of the European Academy of Allergy and Clinical Immunology [5].

The study was performed in accordance with the Declaration of Helsinki and upon obtaining the agreement of the Bioethical Committee of Medical University of Lodz, Łódź, Poland.

\section{RESULTS}

The group consisted of 100 subjects ( 47 females, 53 males). The mean age was $40.6 \pm 7.09$ years. As far as the inclusion criteria are concerned, the most common was vertigo (75\%) (Table 1).

The relations between type I hypersensitivity and certain symptoms, time from exposure to their appearance, as well as time from the last episode were investigated. For this purpose, $\chi^{2}$ and Mann-Whitney U (due to non-normal distributions) tests were used for categorical and numerical variables, respectively. The significance level was set at 0.05 . Allergy to lidocaine was detected in 17 subjects - type I hypersensitivity was diagnosed in 13 cases (skin prick test - 7; intradermic test - 6) and 4 subjects had immuoglobulin $\mathrm{E}$ ( $\mathrm{IgE}$ ) independent allergy (patch test).

In the group where type I hypersensitivity was detected, urticaria $(\mathrm{p}<0.05)$, angioedema $(\mathrm{p}<0.001)$ and rhinitis $(p<0.001)$ were indicated as inclusion criteria more often than among other subjects. The odds ratio estima-

Table 1. The inclusion criteria relating to the occurrence of particular symptoms in the past for the study "Incidence of lidocaine allergy in dentists" conducted in 2012-2016

\begin{tabular}{lll}
\hline \multicolumn{1}{c}{ Symptom } & $\mathrm{n}$ & $\%$ \\
\hline Urticaria & 45 & 45 \\
Angioedema & 40 & 40 \\
Rhinitis & 31 & 31 \\
Dyspnea & 35 & 35 \\
Nausea & 10 & 10 \\
Vertigo & 75 & 75 \\
Fainting & 18 & 18 \\
\hline
\end{tabular}

tion revealed that urticaria increased the odds of detecting type I hypersensitivity 8.57 times. For rhinitis, it was a 10.5-fold increase (Tables 2 and 3).

Similar results were obtained for symptoms after exposure. In this case, the odds ratio of establishing type I hypersensitivity for urticaria was 5.375 , for angioedema - 49.412 and for rhinitis - 15.304 (Tables 4 and 5).

No relation between atopy and type I hypersensitivity was found.

Time from exposure to symptoms appearance was, on average, twice as long as in the group without type I hyper-

Table 2. The relevance between the occurrence of each symptom (inclusion criteria) and detection of type I hypersensitivity in the study "Incidence of lidocaine allergy in dentists" conducted in 2012-2016

\begin{tabular}{lccc}
\hline \multirow{2}{*}{ Symptom } & \multicolumn{2}{c}{ Type I hypersensitivity } & \multirow{2}{c}{$\mathrm{p}(\%)]$} \\
\cline { 2 - 3 } & no & yes & \\
\cline { 2 - 3 } Urticaria & & & 0.03 \\
no & $53(60.92)$ & $2(15.38)$ & \\
yes & $34(39.08)$ & $11(84.62)$ & \\
Angioedema & & & 0 \\
no & $60(68.97)$ & $0(0)$ & \\
yes & $27(31.03)$ & $13(100)$ & \\
Rhinitis & & & 0 \\
no & $66(75.86)$ & $3(23.08)$ & \\
yes & $21(24.14)$ & $10(76.92)$ & \\
Dyspnea & & & 1 \\
no & $57(65.52)$ & $8(61.54)$ & \\
yes & $30(34.48)$ & $5(38.46)$ & \\
Nausea & & & 0.616 \\
no & $79(90.8)$ & $11(84.62)$ & \\
yes & $8(9.2)$ & $2(15.38)$ & \\
Vertigo & & & 1 \\
no & $22(25.29)$ & $3(23.08)$ & \\
yes & $65(74.71)$ & $10(76.92)$ & \\
Fainting & & & 0.707 \\
no & $72(82.76)$ & $10(76.92)$ & \\
yes & $15(17.24)$ & $3(23.08)$ & \\
\hline
\end{tabular}


Table 3. The odds ratio of type I hypersensitivity detection based on the inclusion criteria in the study "Incidence of lidocaine allergy in dentists" conducted in 2012-2016

\begin{tabular}{lccc}
\hline \multicolumn{1}{c}{ Symptom } & OR & $2.5 \%$ CI & $97.5 \%$ CI \\
\hline Urticaria & 8.574 & 2.136 & 57.649 \\
Angioedema & 411443002.286 & 0 & n.a. \\
Rhinitis & 10.476 & 2.901 & 50.071 \\
Dyspnea & 1.187 & 0.334 & 3.882 \\
Nausea & 1.795 & 0.25 & 8.383 \\
Vertigo & 1.128 & 0.311 & 5.365 \\
Fainting & 1.44 & 0.297 & 5.402 \\
\hline
\end{tabular}

Table 4. The occurrence of each symptom after administering the anesthetic and detection of type I hypersensitivity in the study "Incidence of lidocaine allergy in dentists" conducted in 2012-2016.

\begin{tabular}{|c|c|c|c|}
\hline \multirow[t]{2}{*}{ Symptom } & \multicolumn{2}{|c|}{$\begin{array}{l}\text { Type I hypersensitivity } \\
{[\mathrm{n}(\%)]}\end{array}$} & \multirow[t]{2}{*}{$p\left(\chi^{2}\right)$} \\
\hline & no & yes & \\
\hline Urticaria & & & 0.031 \\
\hline no & $43(49.43)$ & $2(15.38)$ & \\
\hline yes & $44(50.57)$ & $11(84.62)$ & \\
\hline Angioedema & & & 0 \\
\hline no & $70(80.46)$ & $1(7.69)$ & \\
\hline yes & $17(19.54)$ & $12(92.31)$ & \\
\hline Rhinitis & & & 0 \\
\hline no & $64(73.56)$ & $2(15.38)$ & \\
\hline yes & $23(26.44)$ & $11(84.62)$ & \\
\hline Dyspnea & & & 1 \\
\hline no & $56(64.37)$ & $8(61.54)$ & \\
\hline yes & $31(35.63)$ & $5(38.46)$ & \\
\hline Nausea & & & 0.364 \\
\hline no & 77 (88.51) & $10(76.92)$ & \\
\hline yes & $10(11.49)$ & $3(23.08)$ & \\
\hline Vertigo & & & 0.747 \\
\hline no & $18(20.69)$ & $2(15.38)$ & \\
\hline yes & $69(79.31)$ & $11(84.62)$ & \\
\hline Fainting & & & 0.728 \\
\hline no & $67(77.01)$ & $11(84.62)$ & \\
\hline yes & $20(22.99)$ & $2(15.38)$ & \\
\hline
\end{tabular}

Table 5. The odds ratio of type I hypersensitivity detection, based on symptoms occurring after administration of anesthetic in the study "Incidence of lidocaine allergy in dentists" conducted in 2012-2016

\begin{tabular}{lccc}
\hline \multicolumn{1}{c}{ Symptom } & OR & $2.5 \%$ CI & $97.5 \%$ CI \\
\hline Urticaria & 5.375 & 1.343 & 36.065 \\
Angioedema & 49.412 & 8.843 & 931.703 \\
Rhinitis & 15.304 & 3.757 & 103.88 \\
Dyspnea & 1.129 & 0.318 & 3.686 \\
Nausea & 2.31 & 0.462 & 9.18 \\
Vertigo & 1.435 & 0.344 & 9.807 \\
Fainting & 0.609 & 0.089 & 2.517 \\
\hline
\end{tabular}

sensitivity $(20.5 \pm 8.23$ vs. $9.85 \pm 2.23, \mathrm{p}<0.001)$. The total number of cases was 89 , as 10 subjects without any symptoms were omitted and 1 missing data record appeared (Figures 1 and 2).

A multiple logistic regression analysis was performed for the presence of type I hypersensitivity as a dependent variable. Initially, the symptoms after exposure - urticaria, angioedema and rhinitis, as well as time from exposure to symptoms appearance and years from the last episode were used as independent variables. The backward stepwise procedure was used to obtain the model presented below. According to the results, the occurrence of angioedema increased the risk of detection of type I hypersensitivity 68.8 times, and extending the period from the last episode by one year decreased this risk by circa $55 \%$ (Table 6 ).

\section{DISCUSSION}

The adverse reactions regarded as allergic can be caused by completely different factors, which in turn can lead to the premature diagnosis of an allergy based only on the medical history or, conversely, to neglecting the symptoms caused by allergic reaction. Individuals whose medical history records indicate such symptoms as urticaria, angioedema or rhinitis, associated with the administration of a local anaesthetic, require detailed diagnostics in an allergy centre. 


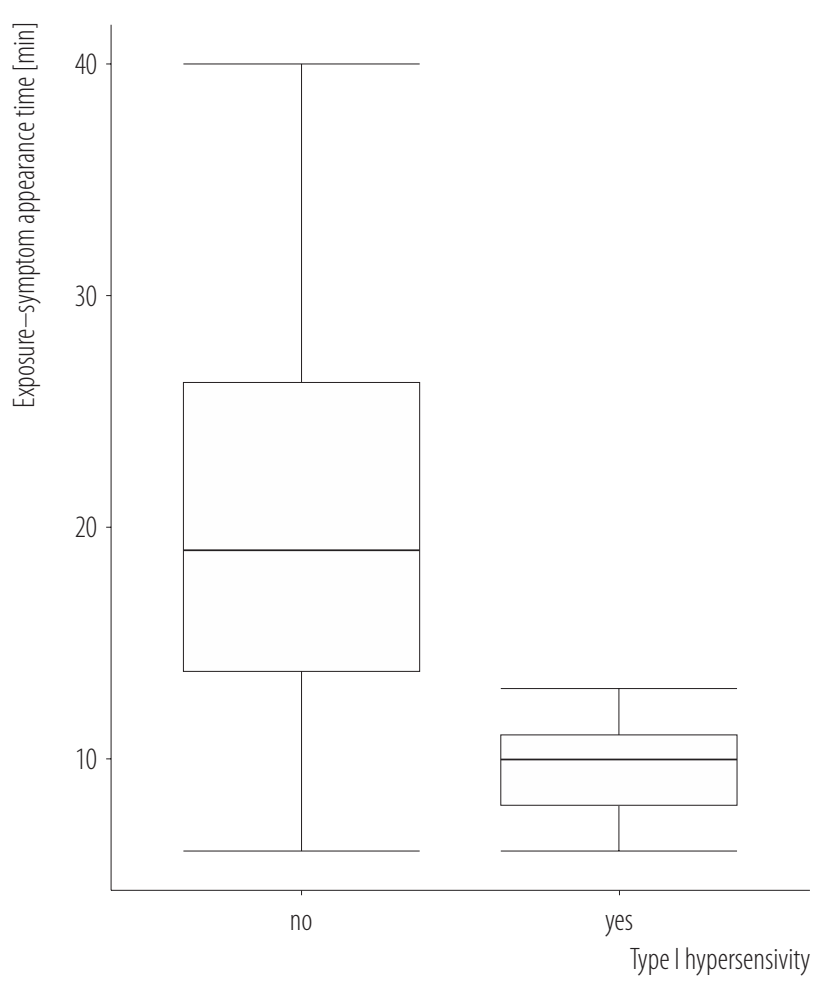

Figure 1. Time from exposure to symptoms appearance and type I hypersensitivity

Such reactions can be directly related to the anaesthetic (allergy reaction) and its dosage (toxic reaction or overdose). In most cases, the adverse reaction after administering the anaesthetic is caused by a psychological effect such as fear and anxiety related to the dental procedure. The literature shows that allergies to local anaesthetics account for $1 \%$ of all adverse reactions, which is similar in incidence with the prevalence of contact allergy to methyl methacrylate [6-8].

In the assessment of adverse reactions after local anesthesia, one must take into consideration also other factors

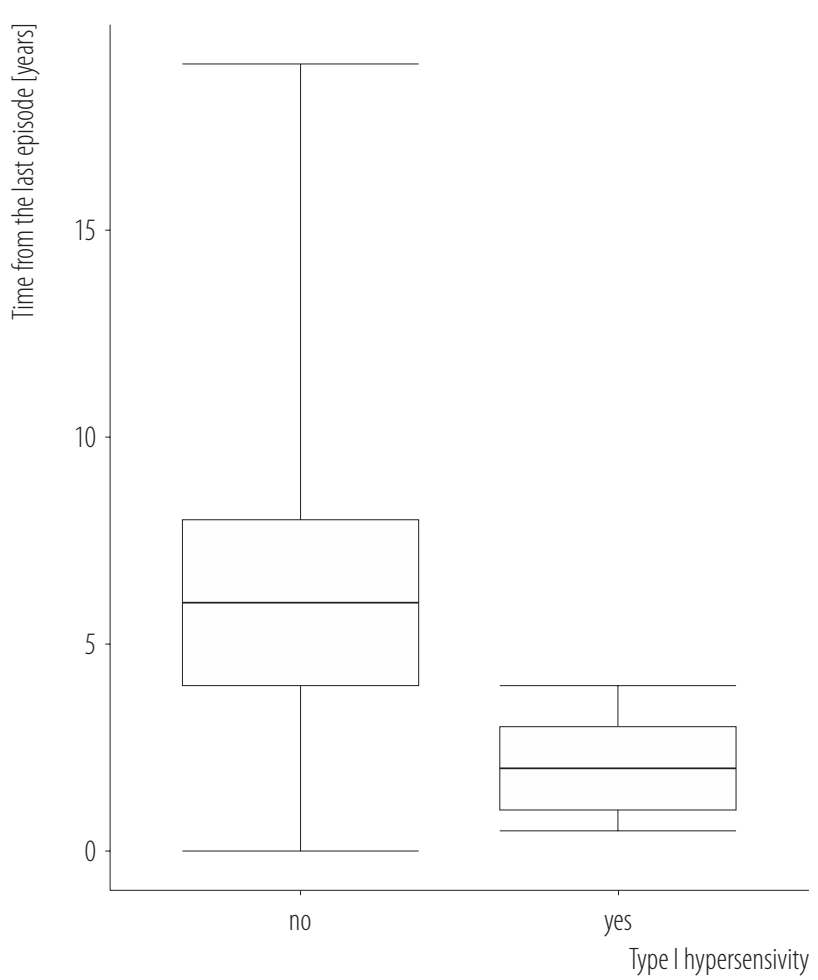

Figure 2. Time from the last episode and type I hypersensitivity

that may resemble allergic reaction. These include toxicity, vasovagal response, incorrect drug administration and the influence of adrenaline, which is often added to the anaesthetic, as well as autonomic or anxiety reactions.

Most toxic effects entail the excessive drug concentration in blood, which is caused by incorrect, intravenous injection, excessive dosage, or impaired function of the body organs responsible for drug metabolism [9-11].

Autonomic reactions, such as vertigo, nausea, excessive perspiration and palpitation, manifest themselves usually directly before a certain procedure is performed. Accord-

Table 6. Multiple logistic regression results in the study "Incidence of lidocaine allergy in dentists" conducted in 2012-2016

\begin{tabular}{lccccccc}
\hline \multicolumn{1}{c}{ Variable } & Estimate & $\mathrm{SE}$ & $\mathrm{z}$ & $\mathrm{p}$ & $\mathrm{OR}$ & $2.5 \% \mathrm{CI}$ & $97.5 \% \mathrm{CI}$ \\
\hline Intercept & -2.12 & 1.064 & -1.993 & 0.0463 & 0.1201 & 0.006448 & 0.6573 \\
Angioedema after anesthetic & 4.231 & 1.314 & 3.221 & 0.00128 & 68.82 & 8.161 & 1837 \\
Time from the last episode [years] & -0.8084 & 0.2929 & -2.76 & 0.005778 & 0.4456 & 0.2204 & 0.7219 \\
\hline
\end{tabular}


ing to other authors, about $22 \%$ of patients that are admitted to the medical facility are developing a stress reaction when anticipating an injection, and $8 \%$ experience anxiety without any specific reason [12].

In rare cases, the occurrence of an autonomic reaction could be an effect of the vasoconstrictors added to solutions of local anaesthetics $[3,4]$.

Severe allergic reaction to a local anaesthetic can resemble the symptoms of a rare disease - the deficiency of $\mathrm{C} 1$ esterase inhibitor, which must be emphasized. It is necessary to add that, according to many authors, symptoms of type I allergic reaction to latex can also be the cause of an individual's being incorrectly diagnosed as allergic to local anaesthetic $[4,13,14]$. The universality of latex products, and their presence in most medical and dental procedures, can lead to developing an allergy in almost everyone. None of the authors, however, has unequivocally commented on routinely performing allergic tests with latex proteins in patients suspected of allergy to local anaesthetics $[4,15]$.

In type I mechanism, the symptoms are reported after $30 \mathrm{~min}$ from administering the drug, but they can also appear after a few hours. In clinical presentation, skin reactions, such as urticaria, severe pruritus and angioedema, are prevalent. They can be accompanied by symptoms from airways, the gastrointestinal tract and the cardiovascular system, leading to anaphylactic shock.

Type IV response is induced most frequently by the contact of the skin with a local anaesthetic, but localized edema can also be caused by an injection [16]. In clinical presentation, delayed reactions are usually visible after $24-48 \mathrm{~h}$, but in the case of local anaesthetics, they can appear even after $2 \mathrm{~h}$ from administering the drug $[4,17]$.

Both types of hypersensitivity can occur in an individual independently. Taking the cross-reactivity under consideration, it is believed that they occur in the same group of compounds - amides and esters. There is, however, a belief that there are no reactions between the esters and amides due to their completely different metabolic pathways $[4,10]$, but, as the literature shows, such allergic reaction has been diagnosed in few patients $[18,19]$.

The results of the presented research indicate that in individuals presenting anaphylactic symptoms in their medical history, such as intensified urticaria, angioedema and rhinitis, the risk of confirming the immunological background of lidocaine hypersensitivity is higher.

Despite the recent advances in allergen characterization and increased awareness of selected allergens, the treatment of occupational allergies can still be improved. Many authors suggest that lidocaine should be included in the European series of basic allergic tests. Testing for hypersensitivity to lidocaine should be considered a periodic examination for general dental practitioners.

\section{CONCLUSIONS}

From the clinical practice point of view, the most important objective was to demonstrate the relation between the medical history indicating the anaphylactic nature of lidocaine intolerance, and an allergy confirmed by skin tests. It is necessary to underline the importance of a thorough medical history in individuals presenting symptoms of local anaesthetic intolerance. A relatively high incidence of positive skin tests in the investigated group, higher than in any other occupational group, is of very high importance.

\section{REFERENCES}

1. Cambell TJ, Williams KM. Therapeutic drug monitoring: antiarrhytmic drugs. Br J Clin Pharmacol. 1998;46(4):307-19, https://doi.org/10.1046/j.1365-2125.1998.t01-1-00768.x.

2. Torsten G, Torsten EG, Lindqvist K. Lidocaine: The origin of a modern local anesthetic. Anesthesiology. 2010;113(6):143377, https://doi.org/10.1097/ALN.0b013e3181fcef48.

3. Moore PA, Van Hersh E. Local anesthetics: Pharmacology and toxicity. Dent Clin North Am. 2010;54(4):587-99, https:// doi.org/10.1016/j.cden.2010.06.015. 
4. Thyssen J, Menne T, Ehlberling J. Hypersensitivity to local anesthetics - Update and proposal of evaluation algorithm. Contact Dermatitis. 2008;59(2):69-78, https://doi.org/ 10.1111/j.1600-0536.2008.01366.x.

5. Heinzerling L, Mari A, Bergmann KL, Bresciani M, Burbach G, Darsow U, et al. The skin prick test - European standards. Clin Transl Allergy. 2013;3(1):3, https://doi.org/ 10.1186/2045-7022-3-3.

6. Corbo M, Wever E, DeKoven J. Lidocaine allergy: Do positive patch results restrict future use? Dermatitis. 2016;27(2):68-71, https://doi.org/10.1097/DER.0000000000 000171.

7. Chowanadisai S, Kukiattrakoon B, Yapong B, Kedjarune U, Leggat PA. Occupational health problems of dentists in southern Thailand. Int Dent J. 2000;50(1):36-40, https://doi. org/10.1111/j.1875-595X.2000.tb00544.x.

8. Syed M, Chopra R, Sachdev V. Allergic reactions to dental materials - A systematic review. J Clin Diagn Res. 2015;9(10):ZE04-9, https://doi.org/10.7860/JCDR/2015/156 40.6589 .

9. Becker DE, Reed LK. Essentials of local anesthetic pharmacology. Anesth Prog. 2006;53(3):98-109, https://doi.org/ 10.2344/0003-3006(2006)53[98:EOLAP]2.0.CO;2.

10. Khan D, Solensky R. Drug allergy. J Allergy Clin Immunol. 2010;2(2):126-137, https://doi.org/10.1016/j.jaci.2009.10.028.

11. Eggleston ST, Lush LW. Understanding allergic reactions to local anesthetics. Ann Pharmacother. 1996;30(7-8):851-7, https://doi.org/10.1177/106002809603000724.

12. Nir Y, Paz A, Sabo EE, Potasman I. Fear of injections in young adults: Prevalence and associations. Am J Trop
Med Hyg. 2003;68(3):341-4, https://doi.org/10.4269/ajtmh. 2003.68.341.

13. Brockow K, Romano A, Blanca M. General considerations for skin test procedures in the diagnosis of drug hypersensitivity. Allergy. 2002;57(1):45-51, https://doi.org/10.1046/j. 0105-4538.2001.00001.x-i8

14. Becker D, Reed K. Local anesthetics: Review of pharmacological considerations. Anesth Prog. 2012;59(2):90-102, https://doi.org/10.2344/0003-3006-59.2.90.

15. Astarita C, Gargano D, Romano C. Long-term absence of sensitization to mepivacaine as assessed by diagnostics protocol including patch testing. Clin Exp Allergy. 2001;31(11):1762-70, https://doi.org/10.1046/j.1365-2222. 2001.01226.x.

16. Breit S, Rueff F, Przybilla B. "Deep impact" contact allergy after subcutaneous injection of local anesthetics. Contact Dermatitis. 2001;45(5):296-7, https://doi.org/10.1034/j.16000536.2001.450510.x.

17. Tomoyasu Y, Mukae K, Suda M, Hayashi T, Ishii M, Sakaguchi M, et al. Allergic reactions to local anesthetics in dental patients: Analysis of intracutaneous and challenge tests. Open Dent J. 2011;5:146-9, https://doi.org/10.2174/1874210 601105010146.

18. Caron AB. Allergy to multiple local anesthetics. Allergy Asthma Proc. 2007;28(5):600-1, https://doi.org/10.2500/aap 2007.28.3035.

19. Berkun Y, Ben-Zvi A, Levy Y, Galili D, Shalit M. Evaluation of adverse reactions to local anesthetics: Experience with 236 patients. Ann Allergy Asthma Immunol. 2003;91(4):342-5, https://doi.org/10.1016/S1081-1206(10)61680-8.

This work is available in Open Access model and licensed under a Creative Commons Attribution-NonCommercial 3.0 Poland License - http://creativecommons.org/ licenses/by-nc/3.0/pl/deed.en. 